\title{
Ruptured Endometriotic Cyst Mimicking Acute Appendicitis During Pregnancy
}

\author{
Ali Özgür ERSOY'1, İrem Eda GÖKDEMIR' ${ }^{1}$, Ebru ERSOY' ${ }^{1}$,ykan YÜCEL ${ }^{1}$ \\ Ankara, Turkey
}

\begin{abstract}
A 21-year-old primigravid pregnant woman of 33 gestational weeks applied to our Perinatology Clinic with acute abdominal pain. The pain was spreading from the midline to the right lower quadrant of the abdomen. Guarding and rebound tenderness existed in the right lower quadrant. An ultrasonographic examination revealed a single alive fetus and normal amniotic fluid. A whole blood count revealed leukocytosis and there were no signs of preterm labor or placental abruption. Fetal heart rate decelerations in a non-stress test were observed, and a decision for cesarean section and abdominal exploration were made.
\end{abstract}

A healthy male baby of 2,500 grams was delivered. In the abdominal exploration, all peritoneal surfaces were coated with a dark red- to brown-colored dense material, like mud. There were two endometriotic cysts in the left ovary; one had a 6-cm mean diameter and was ruptured, while the other was intact and had a mean diameter of 2-3 cm. Both cysts were excised and sent for pathologic examination. The patient had no postoperative problems over two days and was discharged. The final pathologic diagnosis was endometriotic cysts.

Keywords: Pregnancy, Endometriotic cyst, Rupture

Gynecol Obstet Reprod Med 2016;22(2):99-101 DOI: 10.21613/GORM.2016.479

\section{Introduction}

Endometriosis is defined as the presence of functional endometrial tissue outside the endometrial cavity. Endometriosis is a gynecological condition that can cause infertility, chronic pelvic pain, and abdominopelvic adhesions. The incidence is approximately $10 \%$ during reproductive ages (1), however, the exact etiology remains unclear. A wide variety of pathogenic molecular mechanisms exist regarding the pathogenesis. It may be localized to the reproductive system or it may spread to various parts of the body, including the brain and skin (1).

Endometriosis frequently causes clinical dilemmas for gynecologists. Surgery may result in diminished ovarian reserve and the disease may recur despite interventions. Endometriosis surgery for infertility is controversial due to the possibility of diminished ovarian activity. Recent meta analyses support the

\footnotetext{
${ }^{1}$ Department of Obstetrics and Gynecology Zekai Tahir Burak Women's Health Care Training and Research Hospital, Ankara

Address of Correspondence: Ali Özgür Ersoy Zekai Tahir Burak Women's Health Care Training and Research Hospital Ankara, Turkey draliersoy@gmail.com

Submitted for Publication: 28. 01.2015 Accepted for Publication:

30. 03.2015
}

role for endoscopic surgery in chronic pelvic pain (2). Endometriotic cysts rarely occur with pregnancy and its coincidence with pregnancy has been reported as $0.52 \%$ (3).

The present report concerns a case of acute abdominal pain in the third trimester of pregnancy. The aim of this clinical presentation was to discuss this rare clinical condition.

\section{Case Report}

A 21-year-old primigravid pregnant woman of 33 gestational weeks applied to our Perinatology Clinic with acute complaints of abdominal pain, nausea, and vomiting. The pain was spreading from the midline to the right lower abdominal quadrant. The pain had been of a continuous and incremental nature over the previous 72 hours. Prior antenatal surveys revealed no problem. Two doses of betamethasone $(12 \mathrm{mg})$ had been administered for fetal lung maturation. A manual abdominal examination revealed that the patient had guarding and rebound tenderness localized in the right lower abdominal quadrant. An ultrasonographic examination reported a single alive fetus with cephalic presentation and a normal amount of amniotic fluid. The fetal biometry was consistent with 33 weeks of gestation. The placenta was located in the anterior uterine wall and there was no sign of placental abruption in either the physical or ultrasonographic examinations. A nonstress test and cervicovaginal examination revealed no signs 
of preterm labor. The length of the cervical canal was 36 millimeters as measured with transvaginal ultrasonography.

In the differential diagnosis, we investigated appendicitis as a common cause of the acute abdomen. There was no periappendiceal fluid discerned by ultrasonographic examination. Only minimal fluid was seen between the bowel loops. The white blood cell counts were analyzed in 2-hour intervals. The first total leukocyte count was $18,000 / \mathrm{mm} 3$ and there was neutrophil predominance. Two hours later, leukocytosis occurred $(21,000 / \mathrm{mm} 3)$. Serum urea, creatinine, alanine aminotransferase, and aspartate aminotransferase were all within normal limits. An ascending severity of acute abdominal pain, nausea, and vomiting occurred, and a fetal distress pattern was seen with spontaneous decelerations via a cardiotocogram (Figure 1). An emergency cesarean section was subsequently planned.

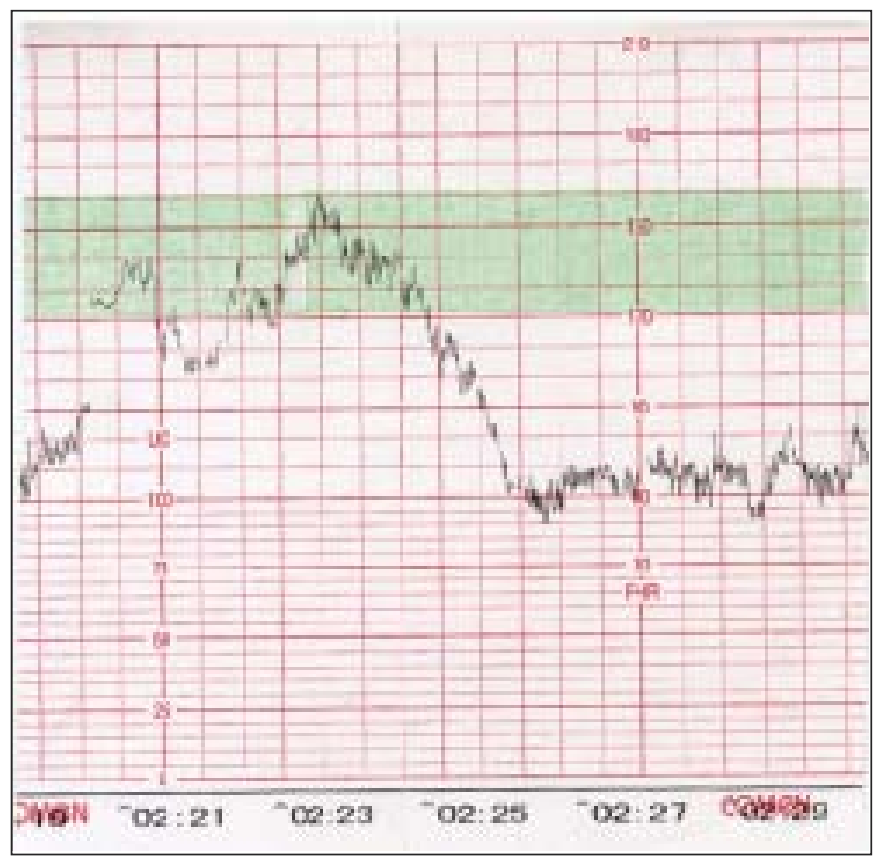

Figure 1: Cardiotocogram depicted the fetal distress pattern

A Pfannenstiel incision was performed in the hypogastric area. A male baby of 2,500 grams was delivered with a lower transverse uterine incision primarily, due to fetal distress. The Apgar score at the first minute was 5, and the Apgar score at the fifth minute was 7. Spontaneous respiration, cardiac activity, and other health conditions of the baby were free of problems without any additional neonatal intervention. The placenta and fetal membranes were removed entirely from the uterus. After uterine suturation and closure, uterine tone was normal. Subsequently, in an open view, all peritoneal surfaces were coated with a dark red, brown, and green, dense material, like mud, as well as some coagulated blood and some fresh blood. The appendix, small and large intestines, right-sided adnexae, and uterus seemed normal. The left ovary had two cystic masses, one of which had been ruptured and shrunken, measuring $6 \times 7 \times 7 \mathrm{~cm}$ in three dimensions, including endometriotic material (Figure 2). The other cyst was intact, measuring $2 \times 3 \times 3 \mathrm{~cm}$ in three dimensions, including endometriotic material (Figure 3). An endometriotic cystectomy was performed by peeling the cyst wall from the ovary and a complete hemostasis was provided by the use of suturation and electrocauterization as low as possible. Excised materials were sent to the laboratory for pathologic examination. The left ovary was reconstructed. All peritoneal surfaces were repeatedly irrigated with $0.9 \%$ isotonic $\mathrm{NaCl}$ solution to prevent chemical peritonitis and adhesions among the intraabdominal viscera. Additionally, there were several millimetric endometriotic foci spreading out to different peritoneal surfaces. Some of these were a dark blue to powder-burn black color as active endometriotic lesions, and some were pale and white colored as inactive lesions with scars (Fig. 4). After two postoperative days, the patient was uneventfully discharged from the hospital and desogestrel (75 $\mu \mathrm{g}$ daily) was prescribed for both the suppression of the other endometriotic lesions and contraception. The drug use was offered after 21 days of puerperium.

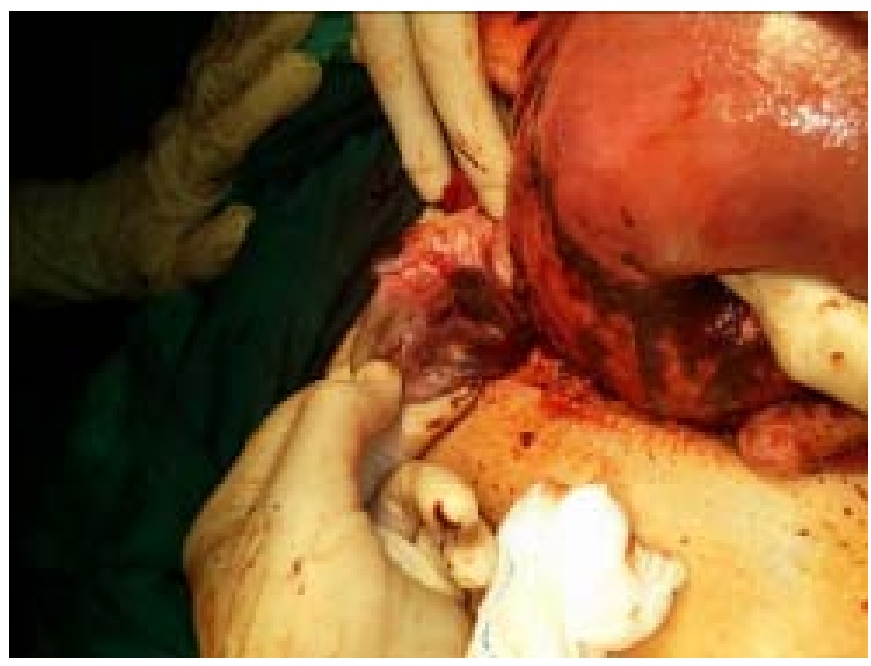

Figure 2: Posterior view of uterus and left ovary which had two endometriotic cysts and one of them had been ruptured.

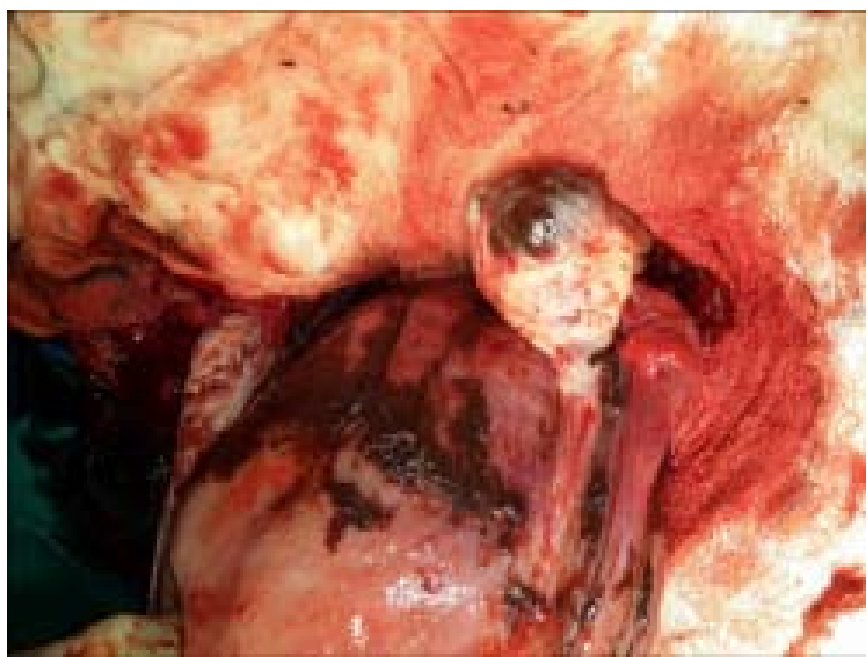

Figure 3: Posterior view of the uterus and the smaller and intact endometriotic cyst in the left ovary can be seen. Note all the peritoneal surfaces was covered by endometriotic material. 


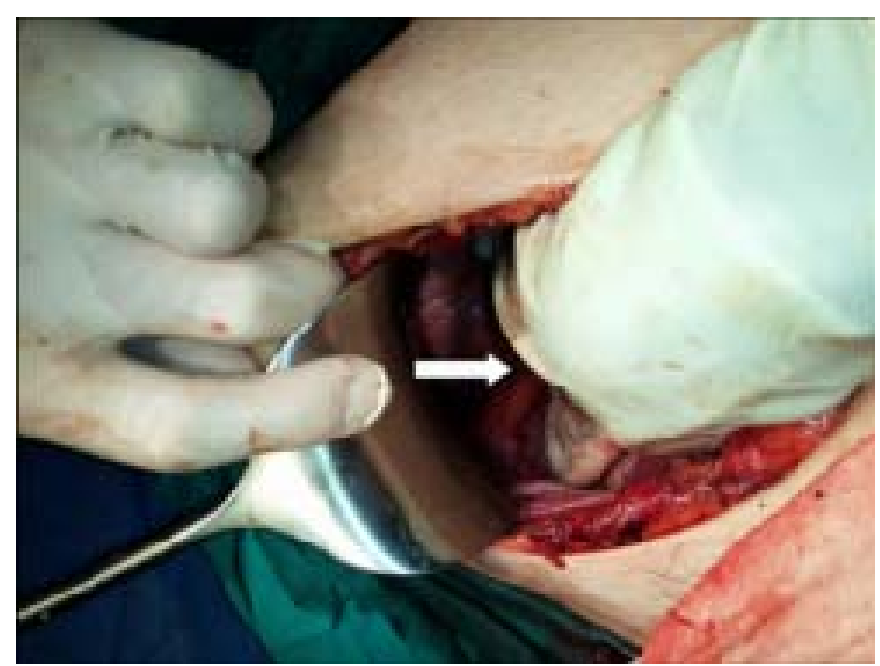

Figure 4: One of several milimetric endometriotic foci spreading to different peritoneal surfaces (white arrow). It can be seen dark-blue, powder-burn black colored and located in front of the right infundibulopelvic ligament.

The pathologic examination was consistent with the clinical diagnosis. Endometriosis was the final pathologic diagnosis for this case and there was no sign of malignancy.

\section{Discussion}

Acute abdominal pain during the third trimester of pregnancy is an uncommon, but potentially serious health condition. The rupture of endometriotic cysts is rarely considered as a cause. The exact incidence of this condition is not clear. The differential diagnosis of acute abdominal pain during pregnancy is frequently a difficult challenge. Interestingly, the rupture of an endometriotic cyst on the left ovary caused pain on the right lower quadrant in our case. We assumed that the preoperative diagnosis was acute appendicitis, as it resembled this condition $(4,5)$.

Only one similar case report of a ruptured endometriotic cyst mimicking acute appendicitis during pregnancy has been reported by Phupong et al. (6). They reported overt leukocytosis similar to our case, but the cause was an infection of an endometriotic cyst, unlike our case. Endometriosis rarely disturbs pregnant women and most gynecologists declare a deactivation of endometriotic lesions during pregnancy (3).

A complete history, physical examination, and an assessment of possible differential diagnoses must be collated to make a diagnosis in a clinical dilemma like this. The differen- tial diagnoses included a uterine rupture, placental abruption, perforated appendicitis, ovarian torsion, urolithiasis, intestinal perforation or obstruction, any form of abscess, and a degenerated or torsioned uterine leiomyoma. Various screening methods may be needed to make the correct diagnosis, in addition to a physical examination. Nevertheless, sometimes none of these methods can help us make the correct diagnosis. In such circumstances, the clinician should take action regarding the emergency signs. For example, he/she could decide on a laparotomy if an acute abdomen persists and the patient's general health conditions are getting worse (7).

The diagnosis of this disease in a pregnant woman presenting with an acute abdomen was difficult; however, this case turned out to be easy because the case required an emergency surgical intervention. Fortunately, antenatal steroid therapy for fetal lung maturation was applied previously as a precautionary measure, and the baby was healthy.

In conclusion, the rupture of an endometriotic cyst should be considered as a possible diagnosis of acute abdominal pain in pregnancy.

\section{References}

1. Holoch KJ, Lessey BA. Endometriosis and infertility. Clin Obstet Gynecol 2010;53:429-38.

2. Duffy JM, Arambage K, Correa FJ, et al. Laparoscopic surgery for endometriosis. Cochrane Database Syst Rev. 2014;4:CD011031

3. Ueda Y, Enomoto T, Miyatake T, et al. A retrospective analysis of ovarian endometriosis during pregnancy. Fertil Steril 2010;94:78-84.

4. Reif P, Schöll W, Klaritsch P, Lang U. Rupture of endometriotic ovarian cyst causes acute hemoperitoneum in twin pregnancy. Fertil Steril 2011;95:2125.e1-3

5. Brosens IA, Fusi L, Brosens JJ. Endometriosis is a risk factor for spontaneous hemoperitoneum during pregnancy. Fertil Steril 2009;92:1243-5

6. Phupong V, Rungruxsirivorn T, Tantbirojn P, Triratanachat $\mathrm{S}$, Vasuratna A. Infected endometrioma in pregnancy masquerading as acute appendicitis. Archives of gynecology and obstetrics 2004;269:219-20.

7. Yalcin OT, Hassa H, Zeytinoglu S, Isiksoy S. Isolated torsion of fallopian tube during pregnancy; report of two cases. Eur J Obstet Gynecol Reprod Biol 1997;74:179-82 